\title{
Resiliencia del docente en situaciones de enseñanza y aprendizaje en escuelas rurales de Perú ${ }^{1}$
}

\author{
Teacher's Resilience in Teaching-Learning Situations in Rural Schools in Peru²
}

\section{Resiliência do docente em situações de ensino/aprendizagem nas escolas rurais no Peru ${ }^{3}$}

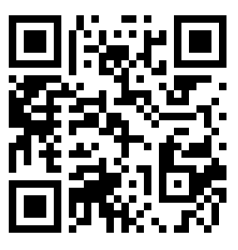

iD

Universidad San Ignacio de Loyola

Lima, Perú

sunquycha2014@gmail.com

https://orcid.org/0000-0003-3589-8706

Universidad Nacional Mayor de San Marcos

Lima, Perú

dfusterg@unmsm.edu.pe

iD https://orcid.org/0000-0002-7889-2243

Yolvi Ocaña-Fernández

Universidad César Vallejo

Lima, Perú

yocanaf@ucvvirtual.edu.pe

https://orcid.org/0000-0002-2566-6875

Recibido • Received • Recebido:03 / 10 / 2018

Corregido • Revised • Revisado: 12 / 03 / 2020

Aceptado • Accepted • Aprovado: 06 / 04 / 2020

\footnotetext{
${ }^{1}$ Este artículo se realizó teniendo como base la tesis"Resiliencia docente en situaciones de enseñanza y aprendizaje en el Perú" para optar el grado de magister en Educación de la Universidad San Ignacio de Loyola, Perú. Con financiamiento del Estado peruano a través del proyecto beca Presidente de la Republica en el año 2015.

${ }^{2}$ TThis article was made based on the thesis "Teaching Resilience in Teaching and Learning Situations in Peru" to opt for the Master's Degree in Education from the San Ignacio de Loyola University, Peru. With financing from the Peruvian State through the President of the Republic scholarship project in 2015.

${ }^{3}$ Este artigo foi elaborado com base na tese "Resiliência de ensino em situações de ensino e aprendizagem no Peru" para optar pelo Mestrado em Educação pela Universidade San Ignacio de Loyola, Peru. Com financiamento do Estado peruano por meio do projeto de bolsa de estudos Presidente da República em 2015.
} 
http://doi.org/10.15359/ree.24-2.20

ROR: https://ror.org/01t466c14 Universidad Nacional, Costa Rica

http://www.una.ac.cr/educare

educare@una.cr

Resumen: El propósito de la investigación es analizar la esencia de la experiencia del docente en situaciones de enseñanza y aprendizaje en escuelas rurales del Perú. La atención a la diversidad de culturas, contextos y estudiantes es complicada; por lo tanto, se requiere de docentes resilientes; es decir, con ciertas características tales como autoestima, autonomía, creatividad, confianza y capacidad de adaptarse a toda circunstancia. La investigación se desarrolló con los parámetros de la investigación cualitativa; específicamente, se empleó la fenomenología hermenéutica en educación, por poseer un carácter reflexivo-emergente. Dentro de esta concepción, se interpreta y comprende el mundo empírico, buscando el significado de las experiencias vividas del personal docente durante sus laborales pedagógicas, con el fin de develar la esencia de estas. Se utilizó la técnica de la entrevista y como instrumento, la anécdota. Participaron cinco docentes: tres varones y dos mujeres; con los siguientes ejes de análisis: expectativa, frustración, tristeza, felicidad y satisfacción. La información se procesó a través de la comprensión de la experiencia, a fin de elaborar significados de un mismo hecho. El estudio concluye que la resiliencia se presenta cuando el personal docente se adapta a diversas situaciones desfavorables presentadas en la escuela, al hacer uso de características personales que le permiten convertir las limitaciones en fortalezas y así superar las adversidades.

Palabras claves: Resiliencia docente; enseñanza y aprendizaje; fenomenología educativa, educación rural.

Abstract: This research aims to analyze the essence of teachers' experience in teaching and learning situations in rural schools in Peru. Attention to the diversity of students, cultures, and social contexts becomes difficult and complicated; it implies having a resilient teacher, with characteristics such as self-esteem, autonomy, creativity, confidence, and ability to adapt to any circumstance. The research used a qualitative design, specifically the hermeneutical phenomenology in education that has a reflexive and emerging character. The empirical world is interpreted and understood within this conception, seeking what the teachers' lived experiences mean in their pedagogical work in which it is intended to unveil the essence of such work. Both the interview as a technique and the anecdote as an instrument were implemented. Five teachers took part in the research, three men and two women. The research had the following axes of analysis: expectation, frustration, sadness, happiness, and satisfaction. The information was processed through the understanding of the experience to elaborate meanings of the same fact. The study concludes that resilience occurs when teachers adapt to various unfavorable situations presented at school, making use of their personal characteristics that allow them to convert limitations into strengths and thus overcome adversity.

Keywords: Teaching resilience; teaching and learning; educational phenomenology, rural education.

Resumo: O objetivo da pesquisa é analisar a essência da experiência como docente em situações de ensino e aprendizagem em escolas rurais do Peru. A atenção à diversidade das culturas, de contextos, e de estudantes é complicada; Por esse motivo, implica a existência de docentes resilientes, ou seja, com algumas características tais como autoestima, autonomia, criatividade, confiança, capacidade de adaptação a qualquer circunstância. A pesquisa foi desenvolvida com os parâmetros da pesquisa qualitativa, especificamente, utilizando a fenomenologia hermenêutica na educação, por ter um caráter reflexivo-emergente. A partir dessa forma, o mundo empírico é interpretado e compreendido, procurando o significado das experiências vividas pelos docentes em seu trabalho pedagógico, que visa revelar sua essência. A entrevista foi utilizada como técnica e a anedota como instrumento, da 
qual participaram cinco docentes: três homens e duas mulheres, com as seguintes linhas de análise: expectativa, frustração, tristeza, felicidade e satisfação. As informações foram processadas por meio da compreensão da experiência, a fim de elaborar significados de um mesmo fato. $O$ estudo conclui que a resiliência surge quando o docente se adapta às diversas situações desfavoráveis que aparecem no centro educativo, fazendo uso de características pessoais que permitem transformar as limitações em pontos fortes e, assim, superar as adversidades.

Palavras-chaves: Resiliência no docente; ensino e aprendizagem; fenomenologia educacional, educação rural.

\section{Introducción}

La educación en el siglo XXI busca desarrollar una sociedad con calidad de vida, para ello necesita dar prioridad a dos de los cuatro pilares de la educación declarados por la Unesco y presidida por Delors (1996): aprender a aprender y aprender a vivir juntos, ya que estos pilares son los cimientos para el cambio educativo. En el estudio, se postula una mirada a las diversas situaciones que, dentro de la escuela, dificultan la labor docente y pueden ocasionar frustración: Al respecto, el Ministerio de Educación (Minedu) no brinda soluciones concretas; por el contrario, tiende a ser oneroso frente al trabajo de docentes en los diversos contextos rurales del país.

La realidad de las escuelas rurales en el Perú es muy crítica, ya que se encuentran marginadas por las políticas del Estado y, lamentablemente, son las que peores resultados obtienen en las evaluaciones nacionales. Cabe señalar que otros países latinoamericanos también enfrentan una realidad parecida a la muestra, tal es el caso en las zonas fronterizas de Costa Rica (SalasAcuña, 2018).

En este contexto, la noción de resiliencia se entiende como la capacidad de sobreponerse y afrontar situaciones difíciles. Cuando el personal docente atraviesa situaciones adversas por diversas razones que inciden en el proceso de enseñanza-aprendizaje, se requiere de esta capacidad de resiliencia para poder enfrentar las dificultades con serenidad, tolerancia, autonomía, entre otras habilidades que deben ser compartidas con el estudiantado.

En los últimos años, la resiliencia desempeña un rol importante en la educación. Según Knakal (2011), la resiliencia en educadores del primer ciclo permite responder con éxito ante la adversidad, al usar las herramientas apropiadas para un aula y autocuidado del propio cuerpo docente.

La necesidad del desarrollo de la resiliencia de docentes en situaciones de enseñanza y aprendizaje en la educación rural es un proceso complejo que inicia en la diversificación curricular como parte del proyecto educativo institucional hasta la concretización de las sesiones de aprendizaje. En la muestra estudiada, cada docente tiene estudiantes de tres y hasta de seis 
grados simultáneamente; proceso enriquecedor de la práctica pedagógica, que requiere que el personal docente se encuentre con una mayor predisposición a realizar un trabajo multinivel en el cual podrá desplegar y desarrollar una serie de capacidades y habilidades para cumplir con los planes propuestos.

La autoaceptación de la adversidad identifica aflicción y padecimiento tácito. En el proceso, se necesita realizar el estudio para comprender dicha experiencia y a partir de ello recomendar aspectos de mejora. Al respecto, Gallego y Cortines (2006), con la experiencia titulada La resiliencia como enfoque pedagógico: Hacia una mirada transformadora del hacer docente, refieren que la singularidad de la experiencia y el hacer de docentes en formación "develan diversos asuntos que tras una reflexión y una práctica crítica se convierten en características y componentes potencializadores" (p. 13). El objetivo es propiciar conductas sanas y constructivas de relevancia social, desde una perspectiva diferente al maltrato o la indiferencia y fortalecer la adquisición de mecanismos de resiliencia en tanto le muestre al estudiantado la relación de la norma con la vida en sociedad (interacción). Estudiar la conciencia, extraer la esencia y sentir las vivencias ayudará a entender la parte interna del sujeto; por ende, es fundamental que nuevas investigaciones indaguen sobre todo ello.

La indagación aspira a que la educación sea socialmente construida, reflexiva y consciente, asumida con voluntad y compromiso pleno; hecho que se realiza en las prácticas educativas, las que deben responder a la demanda social, sin olvidar al individuo como objeto y parte de esta misma, en esta oportunidad a través del método fenomenológico hermenéutico.

La investigación fue realizada con cinco docentes de instituciones educativas unidocentes y multigrados que laboran en el distrito de Mazocruz de la región de Puno, de quienes se recogió la experiencia escrita y luego se entrevistó, con el propósito de poder obtener información relevante acerca de sus desempeños, atendiendo de forma especial los asuntos relacionados con las dificultades afrontadas y situaciones apremiantes, y el cómo han logrado aplicar conductas resilientes a fin de poder revertir situaciones adversas.

\section{Enfoques teóricos de la resiliencia}

El enfoque psicológico del término resiliencia, según lo planteado por Fergus y Zimmerman (2005), aparece en la bibliografía especializada de la salud mental como una hipótesis explicativa de la conducta saludable en condiciones de adversidad o alto riesgo. La resiliencia "supone una combinación de factores personales y contextuales con los cuales el sujeto [afronta] y supera las adversidades en su vida" (Noriega et ál., 2015, p. 45). Diversos estudios han puesto en evidencia que las personas con alta motivación tienen más posibilidades de alcanzar objetivos propuestos, que quienes tienen baja motivación (Kamenetzky et ál., 2009), es decir, enmarcan la importancia de la motivación para el bienestar psicológico. 
En este sentido, Kotliarenco et ál. (1997) acotan que la resiliencia en psicología está afín a la capacidad para presagiar e impedir resultados negativos, a pesar de vivir en escenarios de riesgo constante; así como la recuperación de traumas sufridos. Según Ballesteros et ál. (2006), las distintas perspectivas inciden en el bienestar psicológico. además de establecer una relación entre integración social y salud, así como señalar la importancia de tener puntos de soporte social consistentes como la confianza en la familia, en los amigos, las amigas y en la sociedad para poderse recuperar. Por su parte, Jeong (2019) reconoció que la resiliencia es una idea "que permite la apertura a una visión positiva del abordaje de las problemáticas sociales, donde se profundiza en la prevención desde la promoción de la resiliencia" (p. 68).

El término resiliencia se adaptó a las ciencias sociales para definir a los individuos que, a pesar de vivir en circunstancias adversas, se desarrollan de manera proactiva y significativa en su medio, como parte de un proceso de crecimiento. Entonces, se puede decir que es la capacidad humana que se logra en interacción con el medio ambiente del que la persona sale fortalecida e incluso transformada para reaccionar ante lo negativo de manera productiva (Kotliarenco et ál., 1997). Por todo lo anterior, podemos afirmar que el ambiente familiar, social y cultural favorece el desarrollo de habilidades internas del individuo, lo cual le permite incorporarse al medio contrario en el que se desenvuelve, para continuar recogiendo habilidades sociales como la tolerancia, la confianza, entre otras.

La resiliencia en la filosofía es una mediación reflexiva de confrontar y favorecer recursos para adaptar, afrontar y resarcir lo que el estudiante requiere para su desarrollo personal y social, que conlleve a procesos de profunda reflexión para construir mediación. En ese sentido, cada una de las personas maneja una filosofía de vida que es auténtica y se rige con estos parámetros (Villalta, 2009).

Para Valencia (2007), la resiliencia en la sociología se entiende desde la psicología social, la cual indaga la forma en que los seres humanos se relacionan con otras personas y recalca la influencia de lo social en la conducta humana. En suma, la psicología social aspira a concebir los factores sociales, las relaciones entre los seres humanos y la sociedad en general. Este enfoque sociológico incluye las relaciones familiares, de pareja, con amigos y amigas, y con la sociedad en general, destaca, entonces, la relación entre el individuo y la sociedad.

En la misma línea de pensamiento, Benítez-Corona y Barrón-Tirado (2018) definen la resiliencia:

La competencia del ser humano para hacer frente a los problemas, solucionarlos y salir fortalecido al enfrentarlos; es decir, que, en la circunstancia de sobrellevar la adversidad, el individuo sufre una transformación interna que le da más seguridad en la toma de decisiones benéficas para su vida. (p. 3) 
Según Cortés y Leiva (2012), la resiliencia, en el campo pedagógico, se ubica en el enfoque de la pedagogía crítica y se concibe dentro de la perspectiva de la pedagogía de la reafirmación, la cual presenta tres componentes intervinientes: la refracción, la responsabilidad y la confianza. Dichos factores se ubican en el componente personal y colectivo. Además, la aceptación e impulso personal de integrarse más allá del trabajo colectivo se interponen en campos subjetivos y objetivos aplicados a cada situación.

Asimismo, cobra valor el entorno social que debe afianzar las etapas de reafirmación desde lo personal al encuentro colectivo. La resiliencia pedagógica se establece en el tránsito del proceso educativo, en el cual suceden muchas situaciones difíciles de las que el personal docente se siente comprometido a salir airoso, por lo que busca en la refracción desarrollar la empatía, el autoconocimiento de tener un proyecto de vida sustentable y sostenible. Por otro lado, resalta la responsabilidad como un conjunto de requisitos que en su profesión debe dominar la autonomía, desarrollar el pensamiento crítico e iniciativa para buscar soluciones, con el pilar fundamental de la confianza.

\section{Resiliencia docente}

Actualmente, la resiliencia en educación es considerada como la aptitud de recuperar, recobrar y acomodarse con buena disposición ante cualquier desventura, a fin de desarrollar competitividad en lo social, académico y vocacional, pese a estar en contacto con eventos adversos o estresantes inherentes al mundo de hoy (Gardner y Stephens-Pisecco, 2019). Al respecto, Angulo et ál. (2016) se refirieron a la resiliencia como un "recurso social y psicológico al alcance del hombre para aprender a vivir en medio de las dificultades insoslayables que la vida misma depara" (p. 16). Similar posición es compartida por Gardner y Stephens (2019), quienes formularon que la promoción de la resiliencia en la escuela entre las personas expuestas a diversos factores de riesgo es importante para el desarrollo de una niñez sana física y psicológicamente. Toda persona, sea docente, estudiante, niño, niña, joven, adulta o anciana requiere desplegar la resiliencia, entendida, en educación, como la capacidad de resistir, desarrollar la fortaleza, el coraje para afrontar todos los cambios de la vida en aspectos individuales, familiares y laborales. La palabra resiliente se ha acogido en lugar de fuerte, inmune e indestructible.

Fuentes et ál. (2018) refieren lo siguiente:

La acepción de "resiliente" reconoce el dolor, la lucha y el sufrimiento implícitos en el proceso. El concepto de la resiliencia se aleja del modelo médico del desarrollo humano basado en la patología y se aproxima a un modelo pedagógico proactivo basado en el bienestar, el cual se centra en la adquisición de competencias y eficacia propias. (p. 12) 
Para Rodríguez Soldevilla et ál. (2018), la diversidad de procesos dentro del ámbito educativo está relacionada con una gran variedad de factores que influyen en la formación académica, tales como el desarrollo de las fortalezas y el reconocimiento de las debilidades del estudiantado en general, de forma tal que se puedan resarcir dichas falencias de manera positiva, al buscar que puedan desarrollar mecanismos de resiliencia efectivos para desenvolverse adecuadamente ante situaciones negativas ocurridas en dichos ambientes de aprendizaje.

Es obligatorio examinar el ánimo personal, la constancia, el acometer y el resistir, puesto que toda persona requiere superar sucesos hostiles de estrés, traumas y rupturas en el proceso de vivir, sin quedar marcada de por vida, para su búsqueda de una vida feliz. El resultado es compatible con la pedagogía, pues esta es la ciencia que enseña a vivir bien la vida, a superar momentos dolorosos progresivamente dentro del aula y fuera de ella (Cortés y Leiva, 2012). Por ello se intenta conocer la resiliencia docente en el proceso en mención, para así advertir qué debilidades o fortalezas deben ser erradicadas o afianzadas con mucha paciencia, amor y buen humor, para brindar confianza y creatividad.

Cada docente, en el desarrollo de su labor, atraviesa por diferentes circunstancias negativas y positivas; pero debe tenerse en cuenta cómo sacar lo positivo de cada situación; por lo que, según Panez y Silva (2002), la resiliencia es la "capacidad emocional, cognitiva y sociocultural de las personas o grupos que permiten reconocer, enfrentar y transformar constructivamente situaciones causantes de sufrimiento o daño que amenazan su desarrollo" (p. 18). Por ello, mientras interactúan como educadores y educadoras, los maestros y maestras también pueden ayudar a incrementar la capacidad de resiliencia del estudiantado, al incorporar modelos educativos basados en la fuerza que fomentan la confianza, la motivación y la fortaleza estudiantil (Gardnerd y Stephens-Pisecco, 2019).

Por otro lado, Belykh (2018) ha manifestado que los aspectos relacionados con la aplicación de la resiliencia poseen, en sí, un gran potencial que encamina hacia el empoderamiento de estudiantes dentro del contexto educacional. Por su parte, McKay (2018) manifestó que la resiliencia es necesaria en docentes en proceso de formación, para que se puedan desenvolver como profesionales con buena preparación, capaces de madurar a la par de su profesión; especialmente, en una carrera tan exigente y variada como la enseñanza.

No cabe duda alguna de que aprender a manejar los aspectos emocionales, en especial en situaciones de rigor o crisis, es requerido, sobremanera, en el campo educacional, ya que ello va a repercutir en los comportamientos de sus diversos actores en su interaccionar, tal como lo precisó Nobile (2019):

Problemas asociados al aprendizaje, a la convivencia entre pares y con los docentes, problemáticas entendidas como propias de los adolescentes -como el consumo de sustancias, el embarazo adolescente, la violencia, etc.-, todos ellos podrían ser 
mitigados por la inclusión de propuestas que apuntan al desarrollo en los estudiantes del autoconocimiento y la autoconciencia de las emociones, la regulación emocional, la autonomía emocional y la empatía. (pp. 6-7)

Rodríguez Soldevilla et ál. (2018) realizaron una investigación sobre la vinculación existente entre la resiliencia y el rendimiento académico, en la cual concluyeron que ambos aspectos están relacionados positivamente, pues las relaciones afectivas con los demás permitieron al estudiantado lograr equilibrio frente a las condiciones adversas en el campo académico. Lo anterior, de una u otra manera, va a jugar un rol fundamental en la formación de estudiantes, tal como lo mencionó Nobile (2019):

Es por ello que las formas de interacción así como el tipo de vínculos que se promuevan entre docentes y estudiantes, juegan un rol relevante ya que inciden en la configuración de la experiencia escolar y en la percepción que los estudiantes tienen acerca de su lugar en dicho espacio. (p. 10)

\section{El papel de la adversidad en la resiliencia}

El papel de la adversidad es el inicio de la resiliencia. En la vida diaria se pasa por momentos adversos todos los días, algunos más intensos que otros, y para que se produzca la resiliencia es necesario que estos sucesos adversos sean seguidos de sufrimiento y que esta adversidad haga que progrese el sufrimiento en quien lo experimenta (Trujillo, 2011). Asimismo, los seres humanos con dolor y luego sanación, desgracia y, posteriormente, restauración, van construyendo su capacidad de resiliencia, para ello deben tener algunas características que les ayuden a alcanzar la superación (Cyrulnik, 2002).

En algunas situaciones, atravesamos por sufrimientos que"pueden significar una amenaza para el desarrollo [de la persona]" (Panez y Silva, 2002, p. 19). Esta transformación constructiva nos indica que debemos utilizar todos nuestros recursos para convertir dicha amenaza en un insumo constructivo; es decir, lo negativo convertirlo en algo positivo, sobre todo, tener la tonicidad, flexibilidad y seguridad de los recursos a utilizar. Entonces, la promoción de la resiliencia es fortalecer las capacidades que generan dichos recursos a partir de lo negativo, para que propicien su fácil acceso y utilización a su favor. Este aspecto es lo que otorga a la resiliencia su carácter transformador y enriquecedor de la personalidad (Panez y Silva, 2002).

Por otro lado, el riesgo es considerado como resultado de la convivencia entre la amenaza que se encuentra en el exterior y la vulnerabilidad como algo interno de la persona, que se establece en un momento y lugar determinado. Por ello, el riesgo es considerado como producto de la amenaza exterior y la vulnerabilidad interna. Está latente, constante y oculto, ocasionando a veces crisis ante alguna adversidad de tipo económico, humano o de cualquier 
otra índole. Al ser la amenaza un riesgo externo y la vulnerabilidad algo interno, no todas las personas reaccionamos de la misma manera ante los diversos eventos; por lo cual es necesario desarrollar la resiliencia a lo largo de nuestra historia personal (Cabrejos, 2005).

De la misma forma, cabe mencionar la importancia de los factores o características que posee una persona resiliente. Diferentes estudios coinciden en señalar estos factores como protectores de una persona resiliente, pues ayudan a salir de las adversidades. Algunos de estos son: independencia, introspección, capacidad para relacionarse, iniciativa, humor, creatividad, moralidad y pensamiento crítico; todos son parte del mecanismo interno de cada individuo (Kotliarenco et ál., 1997).

Asimismo, la resiliencia necesita de la creatividad porque es la capacidad innovadora de plantear soluciones novedosas ante situaciones adversas. En estas participan los conocimientos, las emociones y las características de la personalidad e, incluso, el temperamento y carácter en la determinación de algunas decisiones que se necesite tomar. En efecto, se recurre al uso del pensamiento divergente, esto es, imaginar nuevas respuestas ante un problema. Por lo expuesto, la creatividad necesita del pensamiento divergente y los conocimientos, emociones y de la personalidad para poder marcar la diferencia, al permitir ver las situaciones problemáticas desde diferentes ángulos y buscar diferentes soluciones con base en los recursos con los que se cuenta. El problema se asume a manera de reto, a fin de poder solucionarlo (Panez y Silva, 2002).

Igualmente, la autonomía desempeña un rol muy importante, puesto que es la capacidad de decisión personal en un momento dado; la persona toma riesgos que con el paso del tiempo le pueden perjudicar; pero también le puede brindar momentos alegres, ya que el actuar debe ser al instante para no dejar de aprovechar situaciones adversas y convertirlas en positivas. La autonomía se debe forjar con el devenir de las cosas y la historia de vida que cada quien atraviesa; pero, a veces, la persona tiende a esperar la respuesta aprobatoria de sus semejantes, lo cual detiene el proceso de consolidar su autonomía (Panez y Silva, 2002).

De la misma forma, el humor es una característica de la resiliencia referida a encontrarle el lado positivo a lo negativo, es decir, encontrar lo cómico a los problemas, ya sea de manera corporal ofacial, buscando mantener un estado de ecuanimidad para lograr equilibrar los estados emocionales (Fernández-Poncela, 2019). Respecto a la importancia del humor en el desarrollo de la resiliencia en los individuos, se encuentra el trabajo de Losada y Lacasta (2019), quienes hacen una extensa revisión de la bibliografía para referirse al estudio del "sentido del humor como un factor asociado a la Resiliencia" (p. 8). Por otro lado, cabe resaltar la investigación realizada por Moreno de la Cruz y Meneses Eraso (2017), quienes aplicaron mecanismos resilientes basados en el humor a través del teatro en localidades rurales colombianas convulsionadas por la violencia y desidia del Estado. Encontraron que tuvo un efecto significativo, pues "los participantes logran reconocer el humor social como estrategia de afrontamiento efectiva, y desde esa perspectiva 
se generan diversas estrategias de solución ante el fenómeno de violencia" (p.123). Ahora bien, si es que el efecto de la resiliencia catalizada en el humor o favorecida por su desarrollo resulta muy positivo en los individuos, no cabe duda de que su efecto en estudiantes, de forma particular, tenga similares o mejores perspectivas, tal como lo refieren Angulo et ál. (2016):

El humor resiliente representa una dialéctica con posibilidad de convertirse en competencia para fortalecer el espíritu crítico, crear mensajes mediáticos pertinentes, valorar la estética y aprovechar ésta combinación como trampolín para sobrellevar y fortalecer el carácter ante la inevitable llegada de malestares, personales, intramuros y extramuros. p. 14).

Por otro lado, cabe la displicente trascendencia del humor resiliente en el campo educativo, tal como lo expuso Fernández Poncela (2019), al manifiestar que"no cabe duda de la importancia del humor y la risa en la educación, tanto para el alumnado como para el profesorado, por salud física y mental" (p. 61).

Por su parte Angulo et ál. (2016) manifestaron que "hablar de humor resiliente equivale hablar de comunicación; de tan naturales y cotidianas que son ambas, sabemos y entendemos su potencialidad transversal, de manera que el humor resiliente y la comunicación están presentes en todas las disciplinas y profesiones" (p. 18). Aunque pueda otorgársele al humor un estigma negativo dentro de la formación educativa, dicho aspecto no resulta para nada adverso, ya que permite generar un entorno más acorde con estudiantes, al sobrepasar el esquema clásico de la escuela ortodoxa en la cual la rigidez y la sumisión eran sinónimos de respeto. Una actividad académica también pueda ser divertida, tal como lo expresan Bisquerra y Hernández (2017), al manifestar que "el humor no tiene por qué disminuir la disciplina, sino que puede aumentar el respeto hacia el profesor" (p. 60). Similar punto de vista es compartido por Fernández-Poncela (2019), quien establece que el humor permite generar un vínculo más estrecho entre docentes y los estudiantes, cuyos resultados se perciben en la mejora académica y en la generación de un entorno más cordial para los aprendizajes; pero se debe tener en cuenta que debe ser un humor constructivo (Jeong, 2019) o buen humor. Este se puede integrar cómodamente, pues "sí contribuye al autoconocimiento de capacidades y limitaciones del estudiante, colabora en estimular la comprensión de fracasos y el ánimo de remontar problemas, y finalmente, desarrolla la capacidad de tolerar errores y contratiempos, y su afrontamiento con buen ánimo" (Fernández Poncela, 2019, p. 7 0).

\section{La resiliencia y la identidad cultural necesarias para la educación}

Panez y Silva (2002), dentro del modelo de resiliencia, consideran la identidad cultural como un elemento de ella. En la actualidad, se empieza a estudiar la resiliencia desde un enfoque multidisciplinario, considerada tanto un concepto psicológico (características individuales) y un 
concepto sociocultural (familia y comunidad), es decir, es un concepto psicosocial (PROMUDEH, 2001). En momentos en que las prerrogativas pedagógicas refrendan el ideal de la integralidad y contextualización de los aprendizajes, la problemática de la identidad cultural emerge como recurso formativo de incuestionable valía para la elevación de la pertinencia y factibilidad de los proyectos educativos. En virtud de la trascendencia de este axioma, se hace imprescindible el estudio de este fenómeno en su pluralidad conceptual, de modo tal que contribuya a su tratamiento coherente y a su adecuada prospección en el contexto educativo (Chenet Zuta et ál., 2017). Durante el proceso de enseñanza, es imprescindible conocer sobre identidad cultural, ya que por medio de esta nos adentramos al mundo del estudiantado.

La identidad cultural se define como el sentido de pertenencia a una cultura que permite identificar los valorares, creencias, lengua, festividades, danzas, canciones y juegos que van conformando los aspectos culturales de la identidad. Incorporar y recrear las costumbres, idioma, folklore, relaciones sociales, sistema político-económico y educativo del estudiantado le permite ser sujeto activo al personal docente para participar en esa cultura y diferenciarla de los otros grupos con los que les toque trabajar; $y$, si se respeta y parte de esa peculiaridad, se tendrán mejores logros. Este cambio a lo largo del transcurrir de la vida necesita de la colaboración de la familia y otras instituciones como la comunidad (Panez y Silva, 2002).

En tal sentido, el personal docente que conozca su cultura y la respete será resiliente. Vanistendael (1994) propone dos componentes de la resiliencia: En primer lugar, la capacidad de resistencia frente a la destrucción; es decir, la capacidad de proteger la propia identidad bajo presión; y, en segundo lugar, la construcción positiva; es decir, la capacidad para construir pese a las circunstancias difíciles (Puerta de Klinkert, 2002).

\section{Metodología}

La investigación se fundamenta en el enfoque cualitativo, con la metodología fenomenológica-hermenéutica en el área educativa, desde el enfoque desarrollado por van Manen (2003), escasamente desarrollado en América Latina y de forma particular en el Perú. La metodología consistió en la identificación de los significados internos, se enfatizó, constantemente, la idea de la epojé, un concepto que implica el apartar el propio pensamiento para lograr comprender cómo piensa el otro sujeto que, de acuerdo con lo expresado por Villanueva Barreto (2014), se concibe como la supresión de nuestra limitada objetividad, a fin de poder apreciar los hechos por su propia esencia desde una "toma de posición radicalmente distinta respecto de toda objetividad" (Mendoza-Canales, 2018, p. 131). Para ello, los métodos sugeridos por van Manen (2003) son de naturaleza empírica y reflexiva o práctica y meditada; los primeros, orientados al recojo de la experiencia y los segundos intentan examinar y constituir las estructuras fundamentales de la experiencia acopiada. 
Por ser métodos de naturaleza empírica, se sitúan en la búsqueda del material experiencial o experiencia vivida de carácter descriptivo, en este caso tenemos la descripción de experiencias personales, la entrevista conversacional (van Manen, 2003). La entrevista conversacional es aquella en la que se formulan interrogantes y elabora una guía de preguntas a partir del análisis de cada protocolo. En este proceso, no se realizan preguntas preparadas y, por lo general, no es necesario realizar tantas preguntas, solo aquellas necesarias para profundizar lo ya manifestado en la anécdota o narración (Fuster Guillén, 2019) y la observación de cerca a través de anécdotas (van Manen, 2003). La observación de cerca procura acercarse, lo más próximo posible, al mundo vital del individuo observado con el objetivo de llegar a aprehender in situ el significado de la experiencia vivida por él.

Se realizó un muestro no probabilístico por conveniencia, para lo cual se trabajó con cinco docentes del nivel primario del distrito de Santa Rosa, pues la investigación requiere de docentes con características similares y que pertenezcan al mismo ámbito local y grupo social, para generar y comprender las interpretaciones más precisas (Hernández y Mendoza, 2018). Posterior al trabajo de campo, se procedió a aplicar los métodos reflexivos, al analizar y examinar las estructuras como la reflexión temática y lingüística, además de la reflexión mediante la conversación en la búsqueda de las esencias del significado, para lo cual se identificó la naturaleza de este fenómeno, mediante el análisis, la reflexión lingüística y la conversación, teniendo en cuenta la epojé y la reducción en todo el proceso de la investigación (Ayala, 2008).

Para este estudio, en cuanto al procedimiento, se consideró a van Manen (2003) con los aportes de Ayala (2008) y Martínez Miguélez (2008), quienes desarrollan las siguientes fases:

\section{Fase 1: Clarificación de presupuestos}

En la clarificación de presupuestos dentro de la fenomenología, quien investiga inicia su trabajo después de haber realizado una exhaustiva y cuidadosa descripción de sus propios prejuicios. Por severa y estricta que sea una persona, la tradición, la religión y la cultura a la que pertenece pueden ejercer influencia. Por tanto, para asegurar una buena partida y buen proceso, se hace necesario liberarse de todos esos prejuicios para que no afecten lo que puede estar diáfano, para lograr la autonomía del pensamiento de la que la filosofía y la ciencia han presumido (Martínez Miguélez, 2014).

\section{Fase 2: Recoger la experiencia vivida}

Esta segunda fase se desarrolló en dos momentos: en el primero, se inicia la investigación de naturaleza descriptiva con el recojo de la experiencia vivida, esta es la narración de la experiencia personal. Al respecto, algunas maestras y maestros describieron una experiencia en situaciones de enseñanza y aprendizaje, tal como la vivieron. En un segundo momento de esta actividad, se lleva a cabo la ampliación y reescritura de la anécdota, con la elaboración

12 
de la guía de preguntas basadas en el análisis minucioso de la anécdota, para iniciar luego la entrevista conversacional (Ayala, 2008).

\section{Fase 3: Reflexionar acerca de la experiencia vivida}

Esta etapa se centra en la reflexión e interpretación del material recolectado, mediante la comprensión de la experiencia, la cual tiene como etapas: la reflexión macrotemática de los significados, la aproximación holística y sentenciosa, y la reflexión microtemática de los significados con sus cinco etapas: aproximación selectiva de marcaje, aproximación detallada línea a línea, determinación del tema central, expresión del tema central en lenguaje científico y la integración de los temas centrales en una estructura particular (Martínez Miguélez, 2014). En cada una de las etapas debe estar presente la epojé (van Manen, 2003).

\section{Fase 4. Escribir-reflexionar acerca de la experiencia vivida}

Antes de redactar el texto fenomenológico, determinaremos qué nos revela el tema central, qué fue lo que se vivió o lo que se muestra y lo afirmaremos con el lenguaje científico. Después de una reflexión intensa, redactamos el texto fenomenológico acopiando los hallazgos de la investigación, cuyo objetivo fenomenológico-hermenéutico, desde la perspectiva de van Manen (2003), es el de "elaborar una descripción (textual) estimulante y evocativa de las acciones, conductas, intenciones y experiencias humanas tal como las conocemos en el mundo de la vida" (p. 37). A continuación, se muestra, en la Tabla 1, un ejemplo del procesamiento de la información.

Tabla 1: La frustración en los aprendizajes de Vera

\begin{tabular}{|c|c|c|}
\hline Párrafo & Tema & Expresión del lenguaje científico \\
\hline $\begin{array}{l}\text { Párrafo n. }{ }^{\circ} 1 \text {. He visto que no hay } \\
\text { mucho apoyo por parte de los padres } \\
\text { de familia, tampoco de la misma } \\
\text { institución, estamos muy distante, } \\
\text { alejado, está un poco abandonada, a } \\
\text { veces uno se siente atado de manos, } \\
\text { sin poder hacer nada, solamente el } \\
\text { docente ve la forma como laborar } \\
\text { para que nuestros niños salgan bien, } \\
\text { ese es el motivo para que los niños } \\
\text { cada año estén disminuyendo y que } \\
\text { mi persona haya sido destacada el } \\
\text { año } 2010 \text { a otra institución. }\end{array}$ & $\begin{array}{l}\text { La docente se siente } \\
\text { frustrada en la institución } \\
\text { por la falta de apoyo } \\
\text { tanto de padres y madres } \\
\text { de familia, del director y } \\
\text { porque está lejos de todo, } \\
\text { haciendo lo que puede en } \\
\text { su labor. }\end{array}$ & $\begin{array}{l}\text { La frustración como el estado o respuesta } \\
\text { del organismo que se desencadena cuando } \\
\text { la profesora experimenta una devaluación } \\
\text { sorpresiva en la calidad o cantidad de } \\
\text { un reforzador de señales previamente } \\
\text { asociadas de gran magnitud. Este concepto } \\
\text { incluye demoras o impedimento en la } \\
\text { llegada de un estímulo reforzador, como } \\
\text { en Ayupalca, donde la docente se siente } \\
\text { sola y nadie le presta ningún apoyo y su } \\
\text { respuesta implica mecanismos emocionales } \\
\text { de estímulos aversivos y de soledad que } \\
\text { desencadena respuestas de estrés. }\end{array}$ \\
\hline
\end{tabular}

Nota: Elaboración propia. 


\section{Resultados de la resiliencia docente en los aprendizajes}

Quienes trabajamos como docentes debemos tener una buena capacidad de resiliencia, dado que por circunstancias laborales nos toca vivir en diferentes realidades y compartir con personas de diversos caracteres, edades, culturas, creencias, tradiciones. Entre estos y otros aspectos, se deben tener en cuenta en situaciones de enseñanza y aprendizaje en escuelas rurales, las cuales no siempre responden a lo esperado y programado. Es decir, surgen situaciones adversas que salen del control del personal docente y para ello debe buscar mecanismos y estrategias que le ayuden a enfrentarlas, de manera que refuercen su labor y les anime a seguir alcanzando logros.

Existen eventos adversos por los que una persona atraviesa, ya que, si no hubiera sucesos negativos en nuestras vidas, no tendríamos la posibilidad de desarrollar la resiliencia. Conocemos también que si no podemos superar los problemas oportunamente, estos causan desilusión, miedo o desconcierto. Para evitar dichos escenarios, debemos estar preparados para superarlos.

Quedé sorprendido de no encontrar a ningún padre de familia, alumnado, ni el presidente de APAFA, no había absolutamente nadie, me sentí un poco apenado. (Párrafo 2. Miky, UT 2)

Como era una institución unidocente me he sentido un poco mal, chocante, yo mismo era director, docente y todo. (Párrafo 3. Miky, UT 2)

He visto que no hay mucho apoyo por parte de los padres de familia, tampoco de la misma institución, estamos muy distante, alejado, está un poco abandonada, a veces uno se siente atado de manos. (Párrafo 1. Vera, UT 1)

La transformación constructiva de la resiliencia en docentes de zonas rurales es necesaria y esta, en algunas circunstancias, nos lleva a algunos límites, pues no encontrar respuesta a situaciones adversas provoca un desequilibrio emocional que, si se identifica a tiempo, se supera con diversas estrategias, que conviertan la dificultad en un reto, al activar mecanismos de defensa que convierten el problema en oportunidad para el desarrollo de la labor académica y éxito en la vida.

También tenía que poner de mi parte, mis estrategias, que es lo que debo hacer con este niño, como debo lograr, entonces esa programación no me ha respondido, nunca me había pasado en diez años, un niño que se escapaba, no le gustaba leer, ni escribir, ni estar en las clases, pero si le gustaba estar jugando en el patio. (Párrafo 21. Miky o, UT 2) 
Uno tenía que adecuarme de acuerdo a lo que se estaba manejando a nivel regional y eso un poco que te frustra, porque no hay una orientación debida de cómo debemos hallar el problema del contexto, uno tiene que aplicar sus propias estrategias y realicé mi programación anual con el Proyecto Curricular Regional que es un poco más contextualizado y ceñido a la realidad. (párrafo 4. Linda, UT 1)

En el trabajo diario, observamos situaciones de amenaza que vienen del exterior, de otra persona, de la sociedad que provoca frustración momentánea o duradera según el grado de vulnerabilidad. Si el personal docente es resiliente, inmediatamente busca una solución sin llegar a estresarse, encuentra la satisfacción constante. El riesgo se presenta en todo momento, depende de la fortaleza y decisión para que no provoque ninguna crisis emocional. El desarrollo adecuado de la resiliencia conlleva a sobrellevar en una constante reflexión.

He visto que no hay mucho apoyo por parte de los padres de familia, tampoco de la misma institución, estamos muy distante, alejado, está un poco abandonada, a veces uno se siente atado de manos sin poder hacer nada, solamente el docente ve la forma como laborar para que nuestros niños salgan bien. (Párrafo 1. Vera. UT. 1)

El autoconocimiento ayuda a elevar o disminuir la autoestima, la cual es la valoración que tiene cada persona de sí misma y posibilita la formación de la personalidad para potenciar o limitar las reacciones o usar los recursos con los que cuenta para poder afrontar las circunstancias positivas o negativas por las que atraviese. En cuanto a las experiencias de fracaso o éxito, la sociedad evaluará y ratificará que la autoestima esté o no en un nivel considerado para tener reacciones constructivas y aminorar los efectos de las adversidades; sin embargo, si la autoestima del personal docente es baja, conduce a una actuación negativa ante cualquier situación por más que esta sea fácil de resolver. Desarrollar la autoestima favorece el enfrentamiento a la adversidad, le ayudará a tener confianza en todos los aspectos de la vida para llegar a la transformación constructiva día tras día.

Me sentía muy contento de llegar, esperaba ansiosamente encontrarme con los niños y con los padres de familia. (Párrafo 1 Miky. UT 1)

He sido destacada en el año 2010, me he sentido bien fortalecida, animosa del trabajo armónico con mis niños y con todos. (Párrafo 9. Vera. UT 2)

Quien me enseñó eso, yo he tenido que aprender eso en el transcurso que iba trabajando en el desarrollo de las sesiones, las cuales tenían que ser más pertinentes al aula y puedes cambiar estrategias. (Párrafo 9.1. Linda UT 2) 
http://doi.org/10.15359/ree.24-2.20

ROR: https://ror.org/01t466c14 Universidad Nacional, Costa Rica

http://www.una.ac.cr/educare

educare@una.cr

La creatividad en la contextualización de los aprendizajes se pone de manifiesto cuando I personal docente se encuentra ante ciertas situaciones adversas en las que tiene que solucionar antes que se agudice y se salga de control.

Tenía que reprogramar mi trabajo, ya no avancé los temas previstos, he trabajado psicomotricidad, lateralidad, costados y todo lo que es orientación espacial, tenía que aprovechar mediante juegos, había logrado lateralidad y otras habilidades, pero en cuanto a conocimientos todavía no. (Párrafo 15, Armando. UT. 3)

Esta se observa constantemente cuando la comunidad educativa presenta expectativas al trabajo del personal docente innovador y creativo, nos referimos específicamente a las actitudes puestas en práctica en la atención a la diversidad de conductas que estudiantes presentan en respuesta a la enseñanza y al desarrollo de las capacidades. El desarrollo de la creatividad docente se presenta en diversas circunstancias, cuando innova su práctica pedagógica según las exigencias y características de sus estudiantes, y la orienta a dar soluciones haciendo uso del pensamiento divergente.

Me presente, me informe, hice un poco de diagnóstico sobre la escuela cómo funcionaba, cómo era unidocente no había alumnos. Pregunté al presidente y miembros de la comunidad, cómo eran las costumbres ¿cuál era la distancia que los educandos recorrían? ¿Cómo venían? para poder trabajar. (Párrafo 4. Miky UT. 1)

El sentido del humor ayuda a sobrellevar las dificultades que se presentan en el proceso educativo pues incluye estrategias que le ayuden a evitar el estrés. Al final, el personal docente muestra apertura ante lo negativo para encontrar lo positivo en una situación adversa; también aprecia el lado positivo de las situaciones problemáticas para sentirse tranquilo y evitar situaciones de estrés.

Nosotros motivamos: ¡niños buenos días! Cantamos, jugamos y el niño no responde se queda sentadito calladito, no dice nada, otro día sólo se ríe. (Párrafo 9, Miky. UT. 2)

Con ellos, el primer día fue bonito, divertido, incluso hubo chacota, se hace así y le ponen un problema si tú quieres le cambias el problema, o el diagnóstico también evalúan a los niños, cómo le evalúan, qué instrumentos utilizó para sacar los saberes previos y el diagnóstico del medio mismo, de la comunidad misma, se hallevadoy fue bonito al compartir mi programación con ellos. (Párrafo 5, Richi. UT 2)

La identidad cultural desempeña un rol importante en la formación de la resiliencia, puesto que respetarla permite su difusión. Es necesario conocer y respetar la cultura y todo lo 
que trae consigo el estudiantado, en torno a ello es relevante realizar la contextualización de los aprendizajes. Participar activamente de la cultura del niño y la niña fortalece la autoestima y autonomía. La identidad cultural en la resiliencia desempeña una función crucial, porque respeta la peculiaridad de cada estudiante y ello permite resolver situaciones diversas.

Los materiales de la zona son más Significativos porque están en contacto con ellos, lo conocen. (Párrafo 18. Miky UT 3)

He planificado primero lo de la comunidad que estaba en el entorno, sus costumbres, tradiciones, sus animales como cuidaban, en base a esto empecé a trabajar las sesiones de aprendizaje, luego con los materiales que nos entrega el Ministerio. (Párrafo 19. Miky UT 3)

La confianza promociona la resiliencia; no obstante, ello no quiere decir que no existan momentos de vacilación en el desarrollo de la labor. Ante estas situaciones, la confianza es el punto de partida que permitirá manejar mejor las situaciones de riesgo o fracaso, asumir las situaciones difíciles para sobreponerse ante ellas y afrontar con entusiasmo los embates de la vida.

El relacionarse con los demás seres fortalece la resiliencia, ya que el conversar con la sociedad permite fortalecer nuestros lazos para así emanar empatía hacia el prójimo y dar apertura a que se puedan manifestar. La capacidad de relacionarse con los demás individuos es llegar a establecer lazos de empatía y confianza, donde prime el respeto en la relación docenteestudiante, docente-docente y docente-comunidad educativa, cuyas relaciones contribuyan a la educación.

La resiliencia es la capacidad humana de enfrentar las situaciones adversas que se presenten, para ello, debemos desarrollar las características ya mencionadas. La resiliencia propone un nuevo paradigma de desarrollo, ya que no todas las personas crecen en igualdad de condiciones, por lo que se hace énfasis en el potencial humano y se hace un llamado a la esperanza, y a la colaboración de todos y todas para realizar la promoción del cambio social que ayude a mejorar la calidad de vida mediante la resiliencia (Figura 1).

\section{Discusión}

La expectativa en una persona se presenta al iniciar algo nuevo o diferente, donde se siente con ganas de trabajar, ilusionada, con todo el entusiasmo de laborar y dar lo mejor de sí. Esta situación pasa desapercibida y no se aprovecha a favor de la educación, con lo cual se resta motivación en el desarrollo del proceso educativo y más aún en el intento de conocer acerca de la nueva realidad. 
http://doi.org/10.15359/ree.24-2.20

ROR: https://ror.org/01t466c14 Universidad Nacional, Costa Rica

http://www.una.ac.cr/educare

educare@una.cr

Figura 1: Fisonomía general de la resiliencia pedagógica en situaciones de enseñanza y aprendizaje en la educación rural

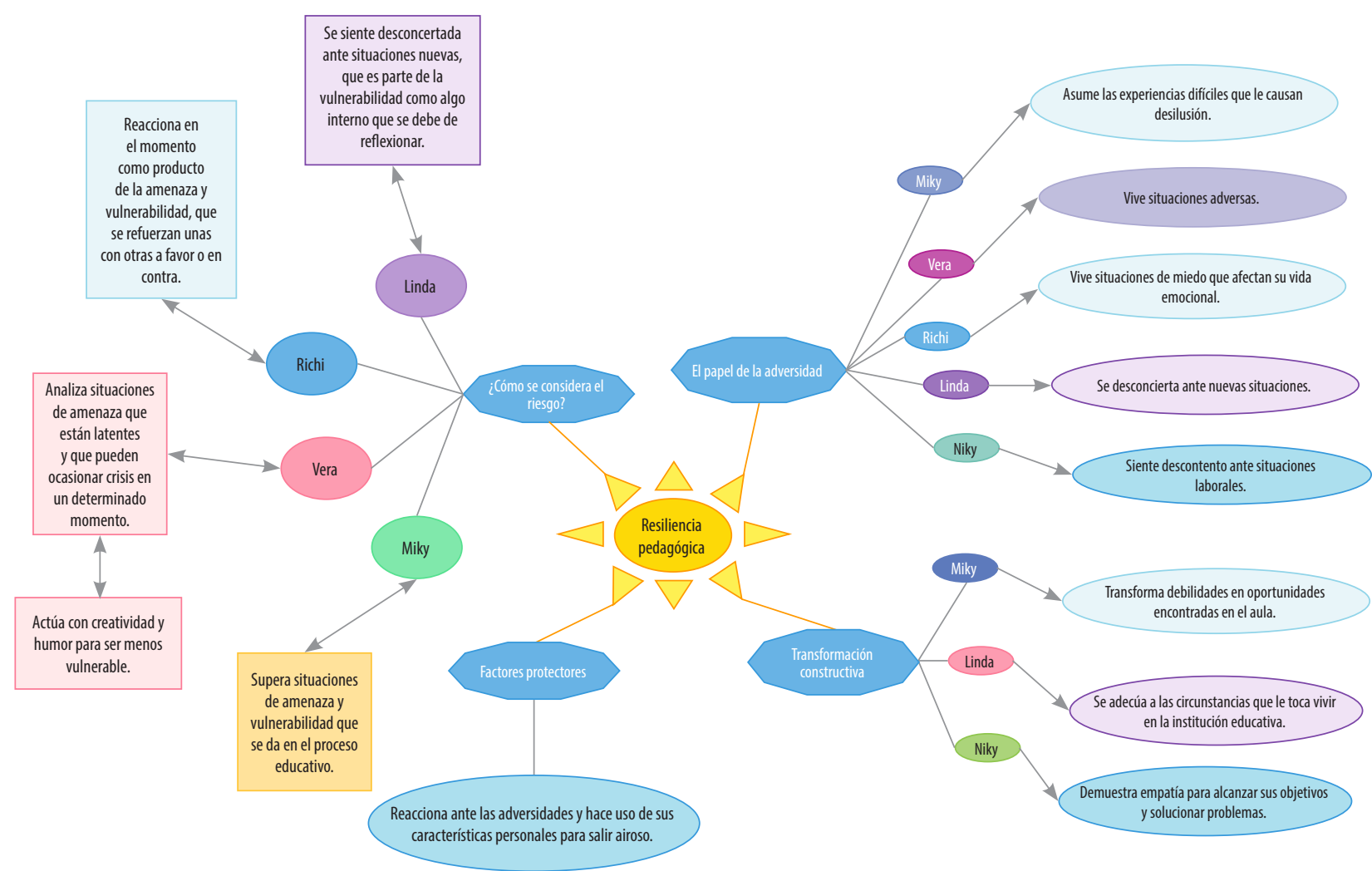

Nota: Elaboración propia.

Es así como, en este estudio se presenta la expectativa en la vivencia de Miky como la reacción ante una circunstancia nueva por la que atraviesa en un momento dado, la cual se relaciona con las experiencias y necesidades por las que ha atravesado y pretende que vuelva a repetir, buscando lo positivo de la situación. Por ello, se puede afirmar que la expectativa puede ser el inicio de la frustración cuando ella es demasiada alta y difícil de concretar, con lo cual se estaría dando inicio al proceso de resiliencia en situaciones de aprendizajes, al llegar el primer día de clases con todo el entusiasmo, donde la expectativa es o se toma como una anticipación o referente de un evento futuro, lo cual se espera que suceda.

Las vivencias transcurridas pueden afirmarse en el proceso de enseñanza aprendizaje. La frustración se da en diversos momentos a lo largo de la labor pedagógica, los cuales no han sido 
tomados en cuenta en los programas oficiales, los cuales reflejan una clara desatención a los detalles y sentir de docentes, así como de estudiantes del ámbito rural. Se desconocen las realidades vivenciales, porque dichos programas solo están orientados al acúmulo de cifras y resultados.

En la presente investigación, se muestra que el personal docente atraviesa por diversas circunstancias y una de ellas es la frustración en situaciones de contextualización, cuando de manera improvista una situación exige más atención, requiere de más recursos de lo previsto y puede generar estrés como es el caso de Miky, quien espera que todo le salga bien, desea cumplir con sus expectativas y, al no cumplirlas totalmente, se siente frustrado, mas aún si se siente solo y desesperado por la falta de apoyo de la comunidad educativa. Al igual que Vera, quien se desconcierta ante situaciones difíciles, por la ausencia de personas a su alrededor con las cuales pueda comunicarse, se siente sola luchando contra la corriente y que debe hacerlo todo como pueda. Así mismo, Linda afirmó que la frustración se produce por la falta de reforzadores del reconocimiento del trabajo realizado y las expectativas que pretende lograr. Siente incertidumbre ante las dificultades que se le presentan y la exigencia de la comunidad que solo piensa en resultados, sin importar por lo que atraviesan docentes y estudiantes. Por ello, la frustración se vuelve el punto de partida para lograr la resiliencia en las instituciones educativas, cuando se llega a superar la frustración y los problemas presentados con actitudes positivas, y sin dificultades no se podría darse dicho proceso.

Como es sabido, cada año se hace una planificación anual al inicio del ciclo escolar para adecuarse a los programas oficiales $y$, al mismo tiempo, responder a las exigencias del estudiantado, teniendo en cuenta las experiencias del año anterior (situación que no siempre se toma en cuenta) sin diversificar los contenidos ni siquiera durante el desarrollo. Por su parte, los grupos docentes, sin percatarse, salen adelante con sus quehaceres educativos gracias a la resiliencia que desarrollan durante el proceso académico, porque la realidad que les toca vivir lo exige; tal como en la investigación lo devela Miky, cuando asume el riesgo como la reacción particular de cada individuo ante la situación que le corresponde vivir y como acciones protectoras a las características o atributos personales con los que uno cuenta, respuesta que le ayuda a sobresalir y reducir daños, considerando siempre el medio en el que se desenvuelve. Desarrolla la resiliencia en momentos de enseñanza y aprendizaje para superar las dificultades de manera significativa y dinámica al cumplir sus metas de modo creativo y proactivo en beneficio propio y de sus estudiantes, ante quienes demostrará seguridad, empatía, creatividad, humor, identidad. Al igual que Linda, que vive la resiliencia al afrontar las dificultades, utilizando sus cualidades personales con perseverancia, identificando potencialidades y debilidades en el medio que se desenvuelve para alcanzar sus metas, las cuales no son estáticas; sino cambiantes.

Es así como la resiliencia se desarrolla en la contextualización de los aprendizajes para poder afrontar las dificultades presentes en dicho proceso. para salir con éxito de este y responder con autonomía, creatividad, introspección, confianza, entre otros. Tal como lo 
sostiene Preciado (2014), la resiliencia es la capacidad de recobrar, superponer y acomodarse con éxito a la adversidad para desarrollar competencias sociales, escolares y vocacionales, pese al estrés que esto signifique.

En la actualidad, la felicidad es un componente de la resiliencia que se vive de manera inconsciente; o sea, no se toma en cuenta con el valor y el realce que tiene, ya que se descuida esta parte importante de la vida escolar y personal, la cual se debe potencializar y vivenciar de manera significativa en el desarrollo del proceso de enseñanza y aprendizaje, por lo que se deben tomar en cuenta las vivencias de la comunidad educativa, tal como se evidencia en este trabajo de investigación, donde Vera vive la felicidad al disfrutar sus logros y demostrar alegría, tranquilidad y bienestar por tiempos más o menos prolongados; pero no definitivos. Por ejemplo, al presentarse dificultades en el desarrollo de la sesión de aprendizaje, como está alegre, puede responder con facilidad, autonomía y seguridad dicha situación y salir de estas con creatividad, viéndolas como una potencialidad y no como debilidad. Dentro de la enseñanza y aprendizaje, la felicidad es parte de la resiliencia, pues brinda estabilidad temporal para volver a iniciar nuevos retos con entusiasmo y serenidad en pro del bienestar tanto personal como estudiantil.

En la educación actual, la tristeza del personal docente y de los grupos de estudiantes nunca es tomada en cuenta, porque son emociones que se quedan en el interior de la persona; sin embargo, se reflejan en el rendimiento profesional y escolar, ya que el estado anímico de la persona influye en su conducta y en el desarrollo de los procesos cognitivos, así como lo muestra esta investigación. Por ejemplo, Richi se siente decepcionado de sus colegas, porque no quieren seguir aprendiendo y se deprime al no alcanza sus metas, lo que influye en su labor pedagógica; pero responde ante cada evento según las circunstancias por las que atraviese y tenga que reflexionar sobre su tristeza para que no le genere cambios o abandono de metas, al caer en una profunda congoja que le puede conllevar a una frustración permanente. De lo anterior se infiere que la tristeza debe ser tratada y no dejar que pase de manera inadvertida, porque si no se atiende a tiempo puede generar frustraciones de manera permanente. Por ello se debe desarrollar la resiliencia para afrontar situaciones difíciles en el proceso de enseñanza y aprendizaje.

La educación del contexto analizado se encuentra muy ajena a los estándares requeridos para ejercer una labor docente adecuada, ya que está carente de procesos de gestión del conocimiento, por lo cual se ralentiza el desarrollo de aprendizajes significativos, pues los programas oficiales no se encuentran adecuados a la realidad rural de la región, situación que revierte en adversidad, y se convierte, quizás, en un detonante para la adquisición de conductas resilientes por parte del personal docente. Así como lo demuestra el presente estudio, que enfatiza a Richi, quien para la llegar a la satisfacción, le presta importancia a las situaciones positivas independientemente del contexto en el que se desenvuelve; disfruta de lo que hace, para mejorar la calidad de vida, y contagia ese entusiasmo al trabajar y así cumple metas 
personales y conjuntas. Por consiguiente, las satisfacciones alcanzadas permiten contagiar entusiasmo a las personas de alrededor, y llegan, así, a cumplir metas, como parte de la resiliencia y las aprovecha a favor de los aprendizajes para que sean significativos y aplicables a la vida del estudiantado. Como lo menciona Veenhoven (1994), le da relevancia preponderante al contexto en el que se desenvuelve la persona para llegar a la satisfacción plena y alcanzar la calidad de vida deseada.

En la actualidad, gran cantidad de docentes que laboran en sectores rurales se sienten sin atención y a la deriva; o sea, en una franca zozobra debido a la desatención de la entidad estatal correspondiente. Dicho aspecto es recogido por el presente trabajo en relación con lo manifestado por Niky, quien ha desarrollado un conformismo al adaptarse a las circunstancias que se le presenten, según su conveniencia para llevarse bien con toda la gente y vivir en comodidad, evitando el estrés. Como resultado, su desempeño docente se ha vuelto rutinario, debido a una desidia producto del conformismo ante la desatención a la cual crónicamente se ve sometido por parte de las autoridades competentes; aspecto que puede revertirse al generarse un adecuado nivel de autoestima y autonomía como parte de su resiliencia para afrontar la contextualización de los aprendizajes y la exigencia venga del él mismo, y no espere seguimiento, sino por convicción y vocación en pro del estudiantado.

La responsabilidad de los grupos docentes se vive de manera constante; pero no consciente, sino por obligación y cumplimiento para no ser sujetos discriminados o juzgados, solo por el hecho de cumplir las horas cronológicas y no horas efectivas en aula y de trabajo intelectual, por ejemplo, así como en las anécdotas acopiadas de la investigación, en la que Niky formaliza lo acordado ante sí mismo y ante los demás en diversas situaciones, siempre reflexionando sobre las consecuencias de sus acciones y cómo afecta a la educación, por lo que debe confiar en sus decisiones y asumir las consecuencias de sus actos, ya que si no contextualiza adecuadamente, los resultados escolares no cumplirán con las expectativas esperadas por las familias y la sociedad. Por ello, la responsabilidad está relacionada con la introspección como característica primordial de la resiliencia y entre ellas se complementan para mejorar el adecuado proceso de contextualización de manera consciente y efectiva.

En el mundo actual, la resiliencia pasa desapercibida ante los ojos de individuos y colectivos; pero el posicionamiento del enfoque fenomenológico-hermenéutico nos devela que la resiliencia se da de manera constante en situaciones de enseñanza y aprendizaje, al afrontar diariamente diversas circunstancia entre docentes, estudiantes y demás miembros de la comunidad que se relacionan directamente, donde cada docente hace uso de los factores protectores ante los riesgos presentados en aula y fuera de ella. Los riesgos en la contextualización vienen a ser todos los inconvenientes que exigen una respuesta inmediata y un esfuerzo, por lo que se puede decir, que es indispensable desarrollar la capacidad de resiliencia en la escuela y la sociedad en general. 
En estos momentos de cambios científicos y tecnológicos, donde la vida transcurre rápidamente, situación que está produciendo estrés hasta el punto de que pueda llegar a convertirse en frustraciones; la actitud del personal docente de aula es un factor esencial para el desarrollo de la resiliencia, ya que llega a movilizar la voluntad y actitud de estudiantes a su cargo, quienes serán resilientes ante cualquier adversidad y no se dejarán vencer por las circunstancias.

En el presente trabajo, la fenomenología permite ver la esencia de los significados que no se perciben directamente, pues están encubiertos por las particularidades de la situación. De acuerdo con lo manifestado, se constató que la resiliencia en la acción pedagógica es un fenómeno experimentado por las educadoras y los educadores, por lo que se convierte en una necesidad para el desarrollo del bienestar humano, por lo que deberá asumirse como una habilidad social ineluctable para poderla aplicar en diversos contextos, aun sean de carácter adverso, a fin de fortalecer la identidad del niño y la niña desde el aula y así se prepare a la niñez y a la juventud para salir airosas ante cualquier dificultad.

\section{Referencias}

Angulo Fuentes, H., Xicoténcatl Valencia, M. L. y Aragón Pérez, V. (2016). Humor resiliente en los productos del universitario de comunicación como competencia para educar en el malestar. Perspectivas Docentes, 61, 14-18.http://revistas.ujat.mx/index.php/perspectivas/ article/view/1855/1505

Ayala, R.(2008).La metodología fenomenológico-hermenéutica de M.van Manen en el campo de la investigación educativa. Posibilidades y primeras experiencias. Revista de Investigación Educativa, 26(2), 409-430. http://www.redalyc.org/articulo.oa?id=283321909008

Ballesteros, B. P., Medina, A. y Caycedo, C. (2006) El bienestar psicológico definido por asistentes a un servicio de consulta psicológica en Bogotá, Colombia. Universitas Psychológica, 5(2), 239-258. http://www.scielo.org.co/scielo.php?script=sci arttext\&pid $=\mathrm{S} 1657-92672006000200004$

Belykh, A. (2018). Resiliencia e inteligencia emocional: Conceptos complementarios para empoderar al estudiante. Revista Latinoamericana de Estudios Educativos, 58(1), 255-282. https://ibero.mx/RLEE/

Benítez-Corona, L. y Barrón-Tirado, M. C. (2018). Análisis cualitativo de resiliencia en estudiantes de posgrado. Revista Electrónica Educare, 22(1), 1-21. https://doi.org/10.15359/ree.22-1.7

Bisquerra-Alzina, R. y Hernández Paniello, S. (2017). Psicología positiva, educación emocional y el programa aulas felices. Papeles del Psicólogo, 38(1), 58-65. https://doi.org/10.23923/pap. psicol2017.2822 
Cabrejos, J. (2005). La promoción de la resiliencia y el diseño de políticas sociales. Revista de la Facultad de Ciencias Económicas Universidad Nacional Mayor de San Marcos, 10(28), 4770. http://sisbib.unmsm.edu.pe/BibVirtual/Publicaciones/economia/numeros/2005 n28. $\underline{\mathrm{htm}}$

Chenet Zuta, M. E.., Arévalo Quijano, J.C.y Palma-Alvino, F. (2017). Identidad cultural y desempeño docente en instituciones educativas. Opción, 33(84), 292-322. http://repositorio.usil.edu. pe/handle/USIL/3219

Cortés, P. y Leiva, J. J. (2012). Resiliencia e interculturalidad en contextos en riesgo de exclusión social: Una perspectiva educativa crítica. En F. Guerra, R. García-Ruiz, N. González, P. Renés y A. Castro (Coords.), Estilos de aprendizaje: Investigaciones y experiencias. V Congreso Mundial de Estilos de Aprendizaje (pp. 1-10), Universidad de Cantabria. https://dialnet.unirioja.es/ servlet/libro?codigo $=555496$

Cyrulnik, B. (2002). Los patitos feos. La resiliencia. Una infancia infeliz no determina la vida. Gedisa.

Delors, J. (Preside). (1996). La educación encierra un tesoro. Ediciones UNESCO.

Fergus, S. y Zimmerman, M. A. (2005). Adolescent resilience: A framework for understanding healthy development in the face of risk. Annual Review of Public Health, 26(1), 339-419. https://doi.org/10.1146/annurev.publhealth.26.021304.144357

Fernández Poncela, A. M. (2019). Educación: Competencias, emociones y humor, perspectivas y estudios. Educación y Humanismo, 21(37), 51-66. https://doi.org/10.17081/ eduhum.21.37.3365

Fuentes, T. E., Mazún, R., Garcilazo, Á. A. y Camargo, O. C. (2018). Identificación de los factores de resiliencia en estudiantes de la carrera de Administración del Instituto Tecnológico Superior Progreso, Yucatán. Advances in Engineering and Innovation, 3(5), 9-17. http:// www.itsprogreso.edu.mx/revistaAEl/index.php/aei/article/view/23/33

Fuster Guillen, D. E. (2019). Investigación cualitativa: Método fenomenológico hermenéutico. Propósitos y Representaciones, 7(1), 201-229. http://dx.doi.org/10.20511/pyr2019.v7n1.267

Gallego, I. T. y Cortines, L. (2006). La resiliencia como enfoque pedagógico: Hacia una mirada transformadora del hacer docente [Tesis de licenciatura]. Universidad de Antioquia, Medellín, Colombia. http://ayura.udea.edu.co:8080/jspui/handle/123456789/1630

Gardner, R. y Stephens-Pisecco, T. (2019). Empowering educators to foster student resilience. The Clearing House: A Journal of Educational Strategies, Issues and Ideas, 92(4-5), 125-134. https://doi.org/10.1080/00098655.2019.1621258 
Hernández-Sampieri, R. y Mendoza Torres, C. P. (2018). Metodología de la investigación: Las rutas cuantitativa, cualitativa y mixta. McGraw Hill

Jeong, K. (2019). Resiliencia y creatividad en escolares de primaria. Revista de Investigación en Psicología, 22(1), 67-78. https://revistasinvestigacion.unmsm.edu.pe/index.php/psico/ article/view/16582/14214

Kamenetzky, G. V., Cuenya, L., Elgier, A. M., Lopez, F., Fosacheca, S., Martin, L. y Mustaca, A. E. (2009). Respuestas de frustración en humanos. Terapia Psicológica, 27(2), 191-201. https:// doi.org/10.4067/S0718-48082009000200005

Knakal, F. (2011). Aporte en el desarrollo de la resiliencia en educadores de primer ciclo del programa HPV, Pudahuel: La mirada de sus profesionales [Tesis de licenciatura]. Universidad Académica de Humanismo Cristiano, Santiago de Chile. http://bibliotecadigital.academia. cl/handle/123456789/2566

Kotliarenco, M. A., Cáceres, I. y Fontecilla, M. (1997). Estado de arte en resiliencia. OPS. http:// bp000695.ferozo.com/wp-content/uploads/2013/02/Resiliencia.pdf

Losada, A. V. y Lacasta, M. (2019). Sentido del humor y sus beneficios en salud. Calidad de Vida y Salud, 12(1), 2-22. http://revistacdvs.uflo.edu.ar/index.php/CdVUFLO/article/ view/172/176

Martínez Miguélez, M. (2008). Epistemología y metodología cualitativa en las ciencias sociales. Editorial Trillas.

Martínez Miguélez, M. (2014). Ciencia y arte en la metodología cualitativa (2.a ed.). Trilllas.

McKay, L. y Barton, G. (2018). Exploring how arts-based reflection can support teachers'resilience and well-being. Teaching and Teacher Education, 75, 356-365. https://doi.org/10.1016/j. tate.2018.07.012

Mendoza-Canales, R. (2018). La fenomenología como teoría del conocimiento: Husserl sobre la epojé y la modificación de neutralidad. Revista de Filosofía, 43(1), 121-138. http://dx.doi. org/10.5209/RESF.60203

Moreno de la Cruz, N. y Meneses Eraso, B. (2017). Teatro popular, una herramienta para fortalecer el humor social frente a la violencia urbana. En Y. A. Gómez Delgado, A. K., Ceballos Mora y O. T., Ruiz Bravo (Comps.), Memoria Congreso Desafíos de la Psicología 2017: Un compromiso con la salud y el bienestar, 123-125. https://doi.org/10.22267/lib.udn.008

Nobile, M. (2019). Introducción: Emociones y afectos en el mundo educativo. Propuesta Educativa, 1(51), 6-14. https://www.redalyc.org/jatsRepo/4030/403061372002/403061372002.pdf 
Noriega Aguilar, G., Angulo Arjona, B. y Angulo Noriega, G. (2015). La resiliencia en la educación, la escuela y la vida. Perspectivas docentes, 58, 42-48. https://dialnet.unirioja.es/servlet/ articulo?codigo $=6349252$

Panez, R. y Silva, G. (2002). Por los caminos de la resiliencia. P\&S Ediciones.

Preciado, S. P. (2014). Fortalecimiento de habilidades para la vida como factores psicosociales en la educación para la resiliencia [Tesis de pregrado]. Universidad Nacional Abierta y a Distancia, Sogamoso, Bocaya, Colombia. https://stadium.unad.edu.co/preview/UNAD. php?url=/bitstream/10596/2758/1/23945691.pdf

PROMUDEH (2001). Manual de intervención infantil para equipos de campo con enfoque de resiliencia en el Ande. Autor.

Puerta de Klinkert, M. P. (2002). Resiliencia. La estimulación del niño para enfrentar desafíos. Lumen.

Rodríguez Soldevilla, A., Díaz Hernández, J. A. y Escamilla Rodríguez, P. (2018). Resiliencia y rendimiento académico en estudiantes de educación superior. Revista Educretam, 1(1), 45-55. http://www.cretam.edu.mx/wp-content/uploads/sites/77/2019/01/revista-nuevoformato-1.pdf\#page $=45$

Salas-Acuña, E. F. (2018). Repensando la educación rural en una zona transfronteriza entre Costa Rica y Nicaragua: El caso de la Región Huetar Norte (RHN). Revista Electrónica Educare, 22(2), 1-19. https://doi.org/10.15359/ree.22-2.18

Trujillo, S. (2011). ¿Hay un origen del proceso resiliente? Una lectura de La maravilla del dolor de Boris Cyrulnik. Psicogente, 14(25), 164-177. http://revistas.unisimon.edu.co/index.php/ psicogente/article/view/1864

Valencia, J. F. (2007). [Reseña del libro Psicología social, por J. F. Morales, F. Moya, E. Gaviria e I.] Cuadrado. Revista de Psicología, 25(2), 339-342. http://pepsic.bvsalud.org/pdf/rp/v25n2/ v25n2a08.pdf

van Manen, M. (2003). Investigación educativa y experiencia vivida. Ciencia humana para la pedagogía de la acción y la sensibilidad. Idea Books. https://es.slideshare.net/kenita/libroinvestigacion-educativa-y-experiencia-de-vida-van-manen

Vanistendael, S. (1994). Resilience: A few key issues. International Catholic Child Bureau.

Veenhoven, R. (1994). El estudio de la satisfacción con la vida. Intervención Psicosocial, 3, 87-116. https://personal.eur.nl/veenhoven/Pub1990s/94d-abs.html 
http://doi.org/10.15359/ree.24-2.20

ROR: https://ror.org/01t466c14 Universidad Nacional, Costa Rica

http://www.una.ac.cr/educare

educare@una.cr

Villalta, M. A. (2009). Factores de resiliencia asociados al rendimiento en estudiantes de contextos de alta vulnerabilidad social. Revista pedagógica, 31(88), 159-190. https:// www.researchgate.net/publication/262476819 Factores de resiliencia asociados al rendimiento academico en estudiantes de contextos de alta vulnerabilidad social

Villanueva Barreto, J. J. (2014). La fenomenología como afirmación de un nuevo humanismo. Universidad Nacional Mayor de San Marcos. http://repositorio.usil.edu.pe/ handle/123456789/1563 